\title{
Three-Dimensional Electrochemical Axial Lithography on Si Micro- and Nanowire Arrays
}

Fedja J. Wendisch, ${ }^{\dagger}$ Michael S. Saller, ${ }^{\dagger}$ Alex Eadie, ${ }^{\dagger}$ Andreas Reyer, $^{\dagger}$ Maurizio Musso, ${ }^{\dagger}$ Marcel Rey, ${ }^{\dagger}$ Nicolas Vogel, ${ }^{\ddagger}$ Oliver Diwald, ${ }^{\dagger}$ and Gilles R. Bourret* ${ }^{*}{ }^{\dagger}$ (1)

${ }^{\dagger}$ Department of Chemistry and Physics of Materials, University of Salzburg, Jakob Haringer Strasse 2A, A-5020 Salzburg, Austria

${ }^{\ddagger}$ Institute of Particle Technology, Friedrich-Alexander University Erlangen-Nürnberg, Cauerstrasse 4, 91058 Erlangen, Germany

\section{Supporting Information}

ABSTRACT: A templated electrochemical technique for patterning macroscopic arrays of single-crystalline $\mathrm{Si}$ micro- and nanowires with feature dimensions down to $5 \mathrm{~nm}$ is reported. This technique, termed threedimensional electrochemical axial lithography (3DEAL), allows the design and parallel fabrication of hybrid silicon nanowire arrays decorated with complex metal nano-ring architectures in a flexible and modular approach. While conventional templated approaches are based on the direct replication of a template, our method can be used to perform high-resolution lithography on pre-existing nanostructures. This is made possible by the synthesis of a porous template with tunable dimensions that guides the deposition of well-defined metallic shells around the $\mathrm{Si}$ wires. The synthesis of a variety of ring

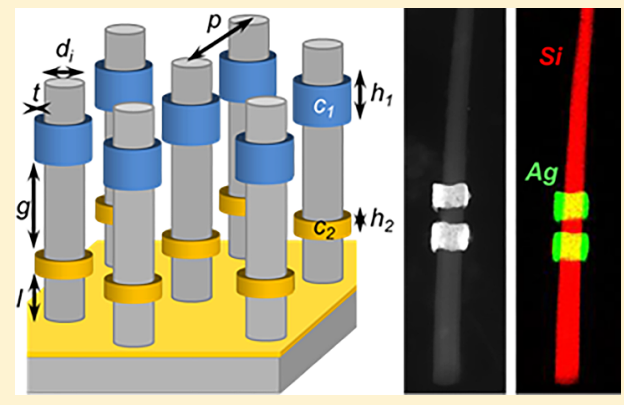
architectures composed of different metals $(\mathrm{Au}, \mathrm{Ag}, \mathrm{Fe}$, and $\mathrm{Ni}$ ) with controlled sequence, height, and position along the wire is demonstrated for both straight and kinked wires. We observe a strong enhancement of the Raman signal for arrays of Si nanowires decorated with multiple gold rings due to the plasmonic hot spots created in these tailored architectures. The uniformity of the fabrication method is evidenced by a homogeneous increase in the Raman signal throughout the macroscopic sample. This demonstrates the reliability of the method for engineering plasmonic fields in three dimensions within Si wire arrays.

KEYWORDS: Si nanowires, lithography, nano-rings, plasmonics, surface-enhanced Raman spectroscopy

S ilicon micro- and nanowires have outstanding tunable optoelectronic properties. $^{1-3}$ Their one-dimensional geometry is particularly attractive because it decouples minority charge-carrier collection from the light-absorption process ${ }^{2}$ while providing a high surface area for catalyst loading., ${ }^{4,5}$ Because Si wire arrays can be easily doped and are compatible with microelectronic fabrication, ${ }^{6,7}$ they have been important candidates for sensors, ${ }^{8}$ photoelectrodes, ${ }^{1,4,5}$ batteries, ${ }^{9}$ photocatalytic systems, ${ }^{10-12}$ solar cells, ${ }^{13}$ nanoscale electronics, ${ }^{6,7}$ and nanoelectrodes for neurological studies. ${ }^{14}$ For most of these applications, the $\mathrm{Si}$ wires need to be properly interfaced with metal nanostructures that can act either as catalysts, ${ }^{4,5,10,11}$ electrical contacts, ${ }^{6,7,14}$ or plasmonic materials. ${ }^{12,15-17}$ Unfortunately, synthesizing hybrid single crystalline $\mathrm{Si}(\mathrm{c}-\mathrm{Si})$ nanowire architectures with defined metal nanostructures is challenging and can only be achieved by performing three-dimensional lithography within the wire arrays, which is not possible with current techniques.

To date, multiphoton microfabrication techniques have been the preferred method for performing lithography in three dimensions. ${ }^{18}$ However, they are usually of low throughput, are not able to produce features smaller than $10 \mathrm{~nm}$, and can only be used to modify polymeric substrates. Various techniques have been used to create complete shells around $\mathrm{Si}$ wires, such as chemical vapor deposition, ${ }^{19}$ electrodeposition, ${ }^{20,21}$ organo- metallic precursor pyrolysis, ${ }^{16}$ or wet chemical synthesis. ${ }^{22}$ Alternative approaches, based on Rayleigh instability ${ }^{23}$ and selective etching of $\mathrm{Si}^{24,25}$ have provided elegant ways to control Si wire morphology. However, these methods are either strictly limited to pure $\mathrm{Si}$ architectures ${ }^{24,25}$ or have constraints in terms of shell composition, dimension, and location ( $>400 \mathrm{~nm}$ range $)^{23}$ that restrict their potential use. Until now, electron-beam lithography has been the only method to pattern metals on Si nanowires. ${ }^{6}$ It is very slow and expensive and cannot be used for patterning three-dimensional nanoscale systems. The ability to locate well-defined metal nanostructures at specific locations within $\mathrm{Si}$ micro- and nanowire arrays could accelerate fundamental studies on hybrid nanostructures and add advanced properties to existing nanowire architectures. For example, recent studies have shown that the position of metal catalysts within nanostructured $\mathrm{TiO}_{2}$ and $\mathrm{Si}$ photoelectrodes can strongly influence photocatalytic activity. ${ }^{5,26}$ Nanometer control over catalyst location within Si nanowire photoelectrodes could aid in the understanding and optimizing of catalyst loading to improve

Received: September 6, 2018

Revised: October 18, 2018

Published: October 25, 2018 
photoelectrode performance. Additionally, because metallic nanostructures can greatly enhance the incident electric field (E-field) under plasmonic excitation, ${ }^{27,28}$ their integration within $\mathrm{Si}$ wire arrays could be used to precisely define plasmonic hot spots in three dimensions within the array. ${ }^{15-17}$ Such enhanced E-fields are of fundamental and technological significance because they can be used to modify important processes, such as light emission and absorption, Raman scattering via the surface-enhanced Raman spectroscopy (SERS) effect, and photochemical reactions via hot electron generation and photothermal effects. ${ }^{15-17,29-37}$ Until now, coaxial lithography (COAL) has been the most versatile technique used to engineer core-shell nanowires. ${ }^{33}$ COAL is built upon the seminal works of Martin, Penner, and Moskovits, who developed electrochemical deposition within porous membranes to synthesize a variety of metal and semiconductor nanowires. ${ }^{38-40}$ Based on the sequential electrodeposition and selective etching of materials with different chemical and mechanical stabilities within porous anodic aluminum oxide membranes, COAL is a powerful method for generating metal shells around nanowires. However, the process is limited to low-quality semiconductor materials that need to be grown from solution, while the straight morphology of the wire and its size and periodicity is fixed and inherited from the rigid alumina template. Additionally, COAL cannot be used to modify pre-existing nanostructures.

Herein, we present a benchtop method, termed threedimensional electrochemical axial lithography (3DEAL), to pattern vertically aligned crystalline silicon micro- and nanowire arrays (diameters ranging from $160 \mathrm{~nm}$ to $1 \mu \mathrm{m}$; Figure 1) with tailored metal architectures. We show the flexibility of the method to coat straight as well as kinked wire arrays with multisegmented metal shells, yielding Si coremetal shell (Si@metal) wires with positive and negative features down to 40 and $5 \mathrm{~nm}$, respectively. The thickness of the metal shells can be controlled in the $30-150 \mathrm{~nm}$ range. The shell can be composed of a variety of metals ( $\mathrm{Au}, \mathrm{Ag}, \mathrm{Ni}$, and $\mathrm{Fe}$ ), while the shell length and position are controlled electrochemically. Using sacrificial shells combined with selective etching, well-defined metal rings around the nanowires can be fabricated. The homogeneity of the structures produced was verified by scanning electron microscopy (SEM), high-angle annular dark field (HAADF) scanning transmission electron microscopy (STEM), and confocal Raman microscopy. We further show that Si nanowire arrays patterned with gold rings show significant enhancement of the Raman signal due to the enhanced electric field around the metal rings.

Square-centimeter-scale $\mathrm{Si}$ wire arrays with well-defined diameters and spacing are prepared using a combination of colloidal lithography ${ }^{41,42}$ and metal-assisted chemical etching (MACE) ${ }^{43-45}$ as is schematically depicted in Figure 1 a. Colloidal lithography is a low-cost technique that combines the large-scale self-assembly of polymeric spheres on planar surfaces (Figures S1 and S2) with a physical evaporation technique, which can be used to generate large-scale gold nanohole arrays. ${ }^{45,46}$ Such gold nanohole arrays can be used to etch silicon via MACE. During the etching process, the metal film sinks into the $\mathrm{Si}$, forming $\mathrm{Si}$ wires. This combined method allows for the preparation of $\mathrm{Si}$ wire arrays with tunable wire diameter, length, and array periodicity (pitch). ${ }^{43}$ After the

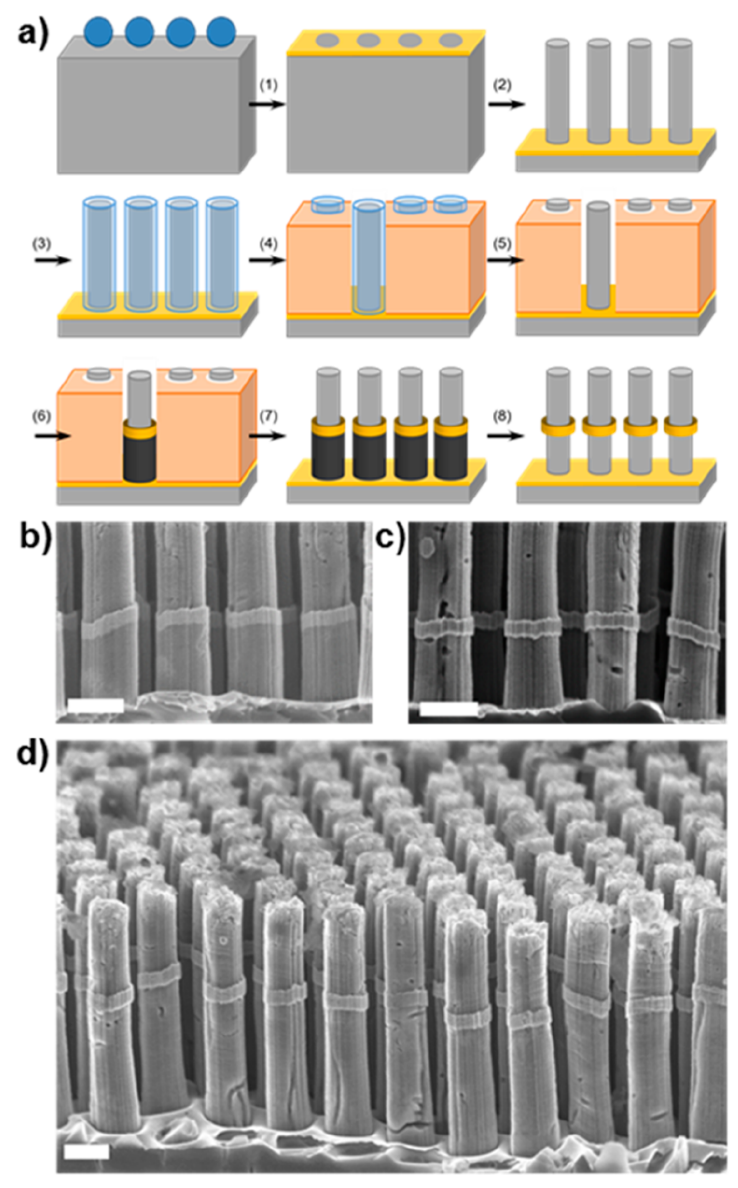

Figure 1. Three-dimensional electrochemical axial lithography within $\mathrm{Si}$ micro- and nanowire arrays. (a) Scheme showing the successive synthetic steps. (1) A loosely packed hexagonal array of polystyrene spheres (in blue) yields a gold nanohole film array (in yellow) on top of the Si wafer (in gray) after colloidal lithography. (2) Si nanowires (in gray) are synthesized via metal assisted chemical etching using the gold nanohole film array as an etching mask. $(3,4)$ Si nanowire arrays (in gray) coated with a $\mathrm{SiO}_{2}$ shell (in blue) are embedded within a polymer film (orange). (5) The dissolution of the $\mathrm{SiO}_{2}$ shell generates annular pores. (6) The pores guide the electrodeposition of multisegmented shells around the $\mathrm{Si}$ nanowires starting from the gold film at the bottom (here, nickel is shown in black and gold in yellow). (7) Dissolution of the polymeric membrane and (8) selective etching of the sacrificial shell (here, nickel) leads to a well-defined metal shell (here, a gold ring). (b-d) Secondary electron SEM images of the various Si-metal microwires synthesized. Scale bars: $1 \mu \mathrm{m}$. (b) $\mathrm{Si}$ wires with a conformal $\mathrm{Ni}-\mathrm{Au}$ shell and (c) after selective etching showing well-defined gold rings. (d) A low-magnification image showing an array patterned with a gold ring with similar dimensions but located at a different position along the wires.

MACE process, the gold nanohole film (used as a mask during chemical etching) is located at the bottom of the wires.

The 3DEAL starts with the synthesis of a porous membrane with tunable dimensions around the Si wires (Figure 1a). The membrane synthesis requires three steps: the coating of the Si wires with a conformal $\mathrm{SiO}_{2}$ shell of tunable thickness grown via a sol-gel process (Figures S3 and S4); deposition of a conformal polycarbonate film to embed the $\mathrm{Si} @ \mathrm{SiO}_{2}$ wires (Figure S5); and selective etching of the $\mathrm{SiO}_{2}$ shell. This leads to the formation of a continuous membrane composed of open annular pores around each $\mathrm{Si}$ wire. At this stage of the synthesis, the nanostructured gold film lays flat at the bottom 
a)

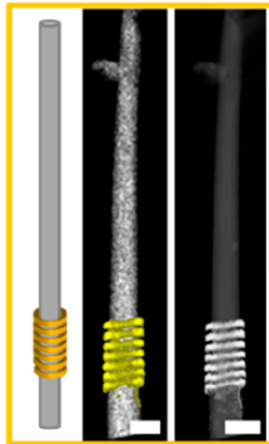

c)

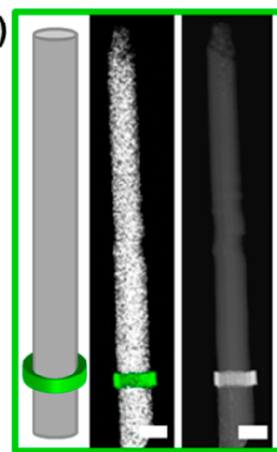

e)
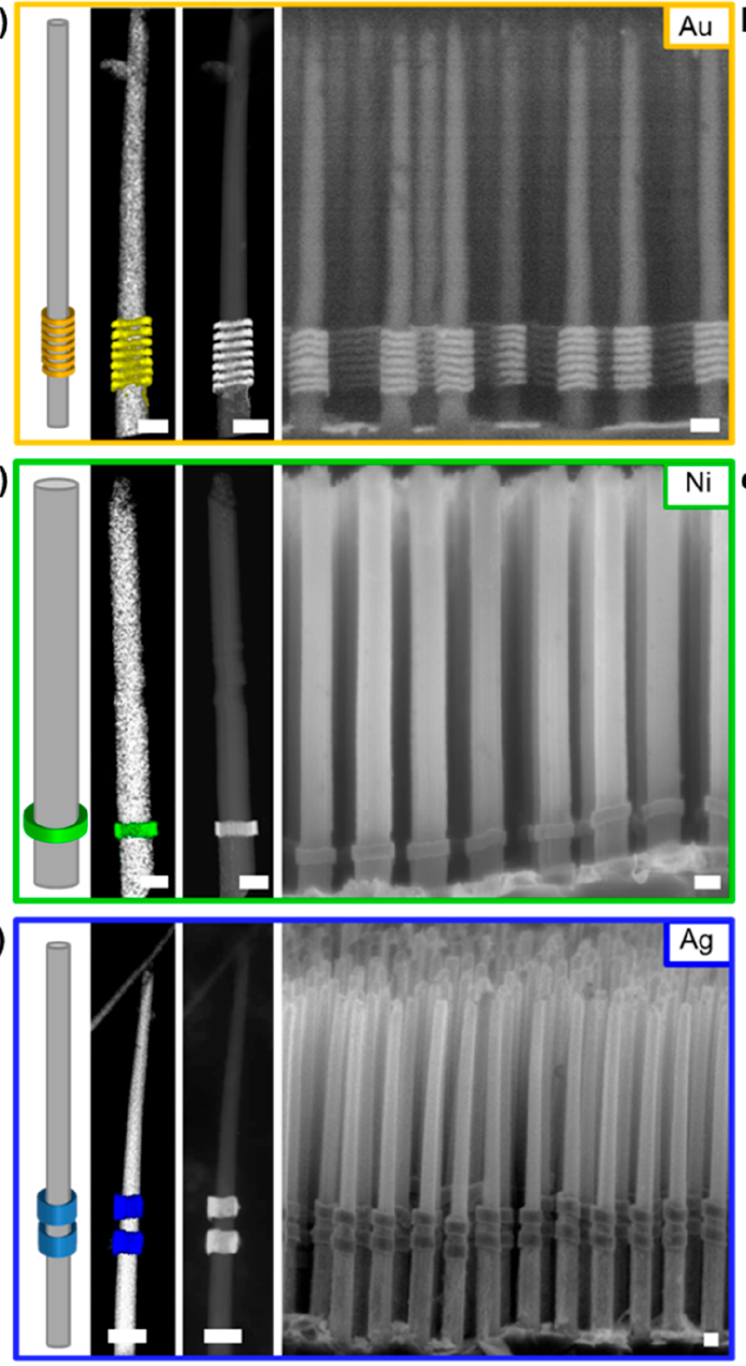
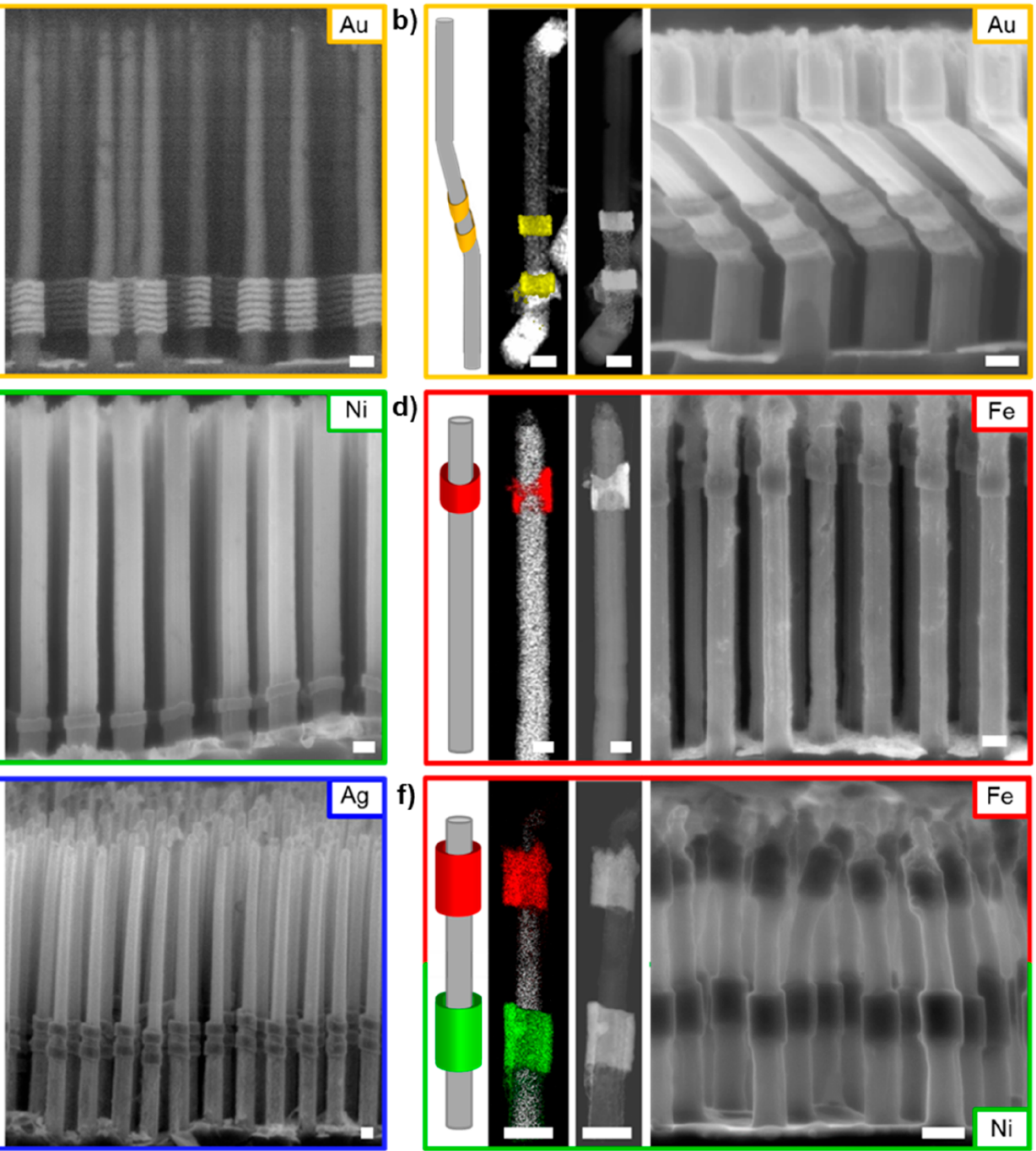

Figure 2. Materials and morphology library of Si@metal nanowire arrays (diameters shown ranging from 160 to $310 \mathrm{~nm}$ with a 590 nominal pitch). All scale bars: $250 \mathrm{~nm}$. (a) Au ring heptamer (gap length: $22 \pm 3 \mathrm{~nm}$ ). (b) Au ring dimer on kinked nanowires. (c) Ni ring located at the bottom. (d) Fe ring located at the top. (e) Ag nano-ring dimers. (f) Fe ring at the top and an Ni ring at the bottom. (a-f) From left to right: structure scheme, STEM elemental map (white, Si; yellow, Au; blue, Ag; red, Fe; and green, Ni), HAADF STEM image, and cross-section SEM images showing (a) backscattered electron signal and $(b-f)$ secondary electron signal.

of the nanowires and can be externally connected to a potentiostat and used as a working electrode. This allows for electrodepositing multisegmented shells around the silicon wires, similar to the growth of metal shells around polymeric cores during COAL. ${ }^{33}$ Prior to the electrodeposition, a thin protective $\mathrm{SiO}_{2}$ shell $(\sim 3-4 \mathrm{~nm}$ thick $)$ is grown around the embedded wire arrays to avoid parasitic growth of metal particles on the silicon wires. EDX analysis shows that the passivating layer present at the $\mathrm{Si}-$ metal interface can be removed with HF after 3DEAL (Figure S6). This result suggests that the electrical contact between the metal and the Si wire should be of acceptable quality. The shell segment thickness (i.e., outer diameter) is controlled by the $\mathrm{SiO}_{2}$ shell thickness, which can be adjusted in increments of ca. $10 \mathrm{~nm}$ (Figures S3 and S7). The shell height depends on the number of coulombs used during electrodeposition, which is set by the deposition time under potentiostatic conditions. ${ }^{32,33,47}$ After dissolving of the polycarbonate, selective etching of the sacrificial shell using appropriate etchant solutions that leave the target material shell intact (Table S2) yields metal rings with well-defined dimensions and positions along the silicon wires (Figure 1).

We demonstrate the synthesis of a variety of metal rings ( $\mathrm{Au}, \mathrm{Ag}, \mathrm{Ni}$, and $\mathrm{Fe}$ ) around $\mathrm{c}-\mathrm{Si}$ micro- and nanowire arrays (Figures 1, 2, and S8-S15) with controlled feature size, spacing, and position along the wires (listed in Table S1). There is no limit to the number and height of rings that can be prepared along the wires. For example, we synthesized Au ring heptamers (i.e., 7 rings) separated by $20 \mathrm{~nm}$ gaps around $\mathrm{Si}$ nanowires (230 nm diameter, Figure 2a). Multimetallic hybrid structures can also be synthesized, such as the $\mathrm{Si} @ \mathrm{Ni}-\mathrm{Fe}$ nanowires shown in Figure $2 \mathrm{f}$, where the Ni ring is located at the bottom of the wire and the Fe ring is at the top. The range of materials compatible with 3DEAL could be extended to metal oxides $\left(\mathrm{TiO}_{2}, \mathrm{ZnO}\right.$, and $\left.\mathrm{MnO}\right)$ and conjugated polymers [polypyrrole, poly(3-hexylthiophene), polythiophene, and poly(3,4-ethylenedioxythiophene] that are chemically stable and can be electrochemically deposited. ${ }^{30,32,33,48,49}$ Less-stable materials could potentially be integrated by using a polyaniline sacrificial shell that dissolves readily in acetone. ${ }^{33}$ Additionally, the metal base layer can be selectively etched: For example, we 

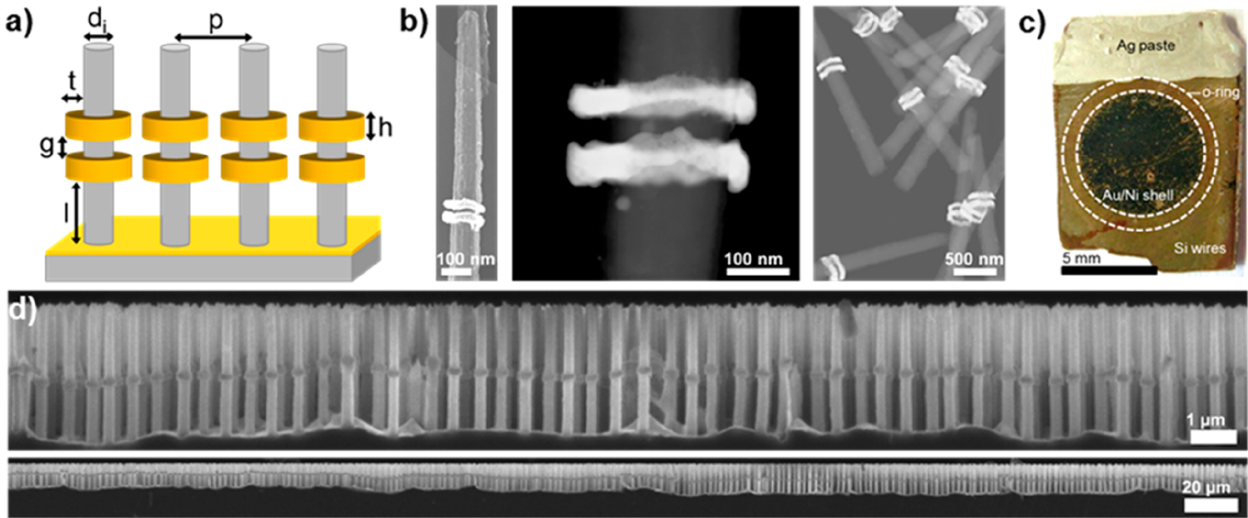

e)

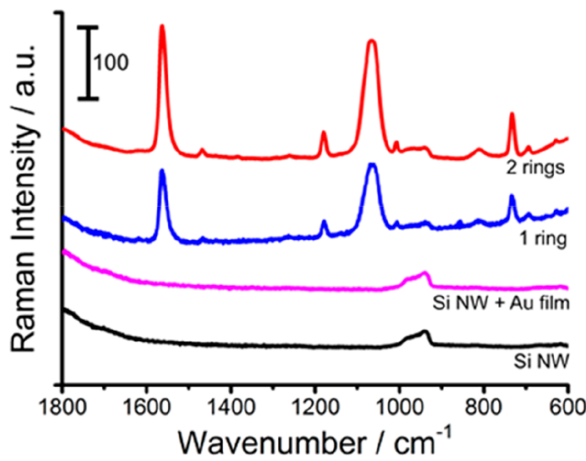

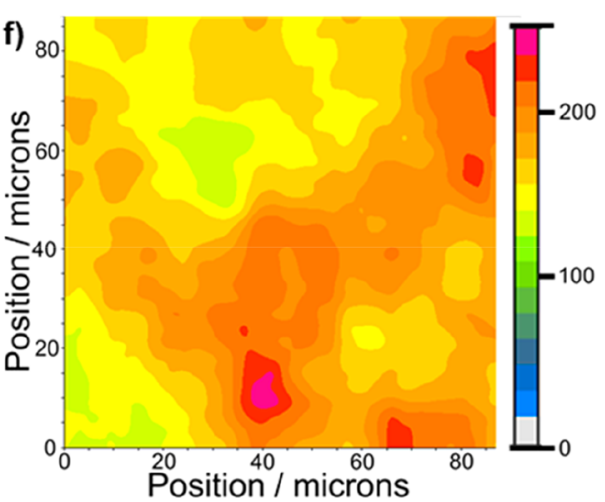

Figure 3. Surface-enhanced Raman scattering on Si@Au nanowire arrays. (a) Schematic showing the geometrical parameters that can be adjusted. (b-d) Arrays patterned with Au ring dimers (30 nm gap). (b) Left: secondary electron STEM image. Center and right: HAADF STEM images. (c) Photograph of the sample after the electrodeposition of the sacrificial Ni and target Au shells, highlighting the large area covered with the hybrid nanowire array. (d) Low-magnification cross-sectional SEM images showing 100\% yield of Si@Au ring dimers. (e) Typical unprocessed Raman spectra after the BDT functionalization of (from bottom to top) Si nanowires (black curve), native Si nanowires with a gold film at the bottom (magenta curve), a single-ring array (blue curve), and a $30 \mathrm{~nm}$ gap ring dimer array (red curve). The spectra are offset for clarity. (f) Smoothed two-dimensional Raman map at $1563 \mathrm{~cm}^{-1}$ (10× objective, NA of 0.25 , and step size of $3 \mu \mathrm{m}$, corresponding to 900 spectra over a $87 \mu \mathrm{m} \times 87 \mu \mathrm{m}$ area), showing that the average signal is homogeneous with a mean value of $177 \pm 23$ counts per second.

prepared Si@Ni wire arrays without a metal base layer by selectively etching the gold film in a $\mathrm{KI} / \mathrm{I}_{2}$ aqueous solution (Figure S16). Because MACE can be performed with a variety of metals, $^{44}$ it should be possible to find an appropriate combination of base layer metal and selective etchant for a specific ring target material. Unlike coaxial lithography that is limited to the modification of straight wires, ${ }^{33}$ our method can be used to pattern kinked nanowires (Figure 2b), which can be produced via a modified version of MACE. ${ }^{50}$ The gap length between the rings can be reliably controlled down to ca. $20 \mathrm{~nm}$ over large areas (Figure 2a), and the smallest negative feature achieved to date is a $5 \mathrm{~nm}$ gap synthesized between two gold rings (Figure S9); however, at these small dimensions, a significant fraction (i.e., > 50\%) of the dimers were bridged, presumably due to local electrode inhomogeneities. The gap length and ring height dispersity is in the $\sim 15 \%$ range, which is typical for such electrochemically controlled processes. $^{30,31,33,47}$ Atomic force microscopy (AFM) measurements show that the Au shell surface is quite smooth (Figure S17) and has a root-mean-square roughness of $0.6 \mathrm{~nm}$. Such a low roughness is most likely inherited from the smooth surface of the templating sacrificial $\mathrm{SiO}_{2}$ shell (Figures S3 and S4), which is properly transferred into the polycarbonate membrane after etching. This suggests that the Si@metal structures will be great candidates for plasmonic applications that require smooth metal films to obtain high-quality surface plasmon resonances. $^{51}$
We demonstrate the ability of the technique to generate optically active nanostructures within crystalline two-dimensional silicon nanowire arrays by patterning arrays with single gold rings and gold ring dimers and characterize them via confocal Raman microscopy (Figure 3). The fast Fourier transform of an SEM image acquired at intermediate magnification shows that the $\mathrm{Si} @ \mathrm{Au}$ arrays prepared are crystalline and hexagonally packed (Figure S15). A total of four samples were prepared using the same Si nanowire array batch (nominal array pitch of $590 \mathrm{~nm}$, nanowire diameter of 195 $\mathrm{nm}$, and height of $\sim 3 \mu \mathrm{m}$ ): native $\mathrm{Si}$ nanowire arrays as produced after MACE with a gold film at the bottom of the wires, Si nanowire arrays prepared by etching the gold film in a $\mathrm{KI} / \mathrm{I}_{2}$ solution, $\mathrm{Si}$ nanowire arrays patterned with single $\mathrm{Au}$ rings (Figure S14), and Si nanowire arrays patterned with gold ring dimers (Figures $3 \mathrm{~b}$ and S15). The rings had an average height of $\sim 63 \mathrm{~nm}$ and thickness of $\sim 47 \mathrm{~nm}$. The gap length between the two rings for the dimer was $\sim 30 \mathrm{~nm}$. The photograph shown in Figure $3 c$ demonstrates that the $\mathrm{Au} / \mathrm{Ni}$ multisegmented shell deposition used to prepare the Au ring dimer sample is homogeneous over macroscopic dimensions $\left(\sim 38 \mathrm{~mm}^{2}\right)$. 1,4-Benzenedithiol (BDT) was used as the SERS target molecule due to its high affinity for gold surfaces and its well-defined Raman peaks. ${ }^{31,36}$ Raman measurements were performed by focusing a $785 \mathrm{~nm}$ laser $(2.5 \mu \mathrm{m}$ diameter, $1 \mathrm{~mW}$ power, $3 \times 20 \mathrm{~s}$ exposures) on top of the $\mathrm{Si} @ \mathrm{Au}$ arrays, expected to irradiate an average of $16 \mathrm{Si@Au} \mathrm{wires.} \mathrm{Noble-}$ 
metal nanostructures can dramatically increase the incident and scattered E-field in the visible near-infrared range. ${ }^{27,28}$ This can lead to large enhancement of the Raman signal, which approximately scales as $|E|^{4}$, where $E$ is the electric field at the metal surface. ${ }^{34} \mathrm{Si}$ nanowire arrays with and without a nanostructured gold film showed no measurable Raman signal, apart from the peaks between 900 and $1000 \mathrm{~cm}^{-1}$ that are due to the second-order Raman scattering of Si (Figure 3e). ${ }^{52}$ The samples containing Au rings, however, showed a large Raman signal, which was highest for the Au ring dimers (Figure 3e). The homogeneity of the Au ring dimer sample was investigated by performing a Raman map over an area of $\sim 7.5 \times 10^{3} \mu \mathrm{m}^{2}$ ( $3 \mu \mathrm{m}$ step size, 900 spectra) by extracting the Raman signal of the $1563 \mathrm{~cm}^{-1}$ peak $(\mathrm{C}=\mathrm{C}$ stretching vibration of the benzene ring structure). ${ }^{53}$ We found an average signal of $177 \pm 23$ counts per second, corresponding to a relative standard deviation of $13 \%$ (Figures $3 \mathrm{f}$ and S18). Considering the complexity of the hybrid structures and the fact that SERS is highly sensitive to minute structural changes, the signal showcases remarkable uniformity. Full three-dimensional electromagnetic simulations, using the finite difference time domain (FDTD) method, suggest that the maximum near-field intensity enhancement at the $785 \mathrm{~nm}$ laser excitation wavelength is generated at the $\mathrm{Si}$ wire-Au ring interface and is around $\sim 780$ and $\sim 930$ for the single $\mathrm{Au}$ ring and the $\mathrm{Au}$ ring dimer, respectively (Figure 4). For simplicity, only one Si@Au nanowire was modeled (see Figure S19 for the full maps). Assuming that most of the Raman signal at a $785 \mathrm{~nm}$
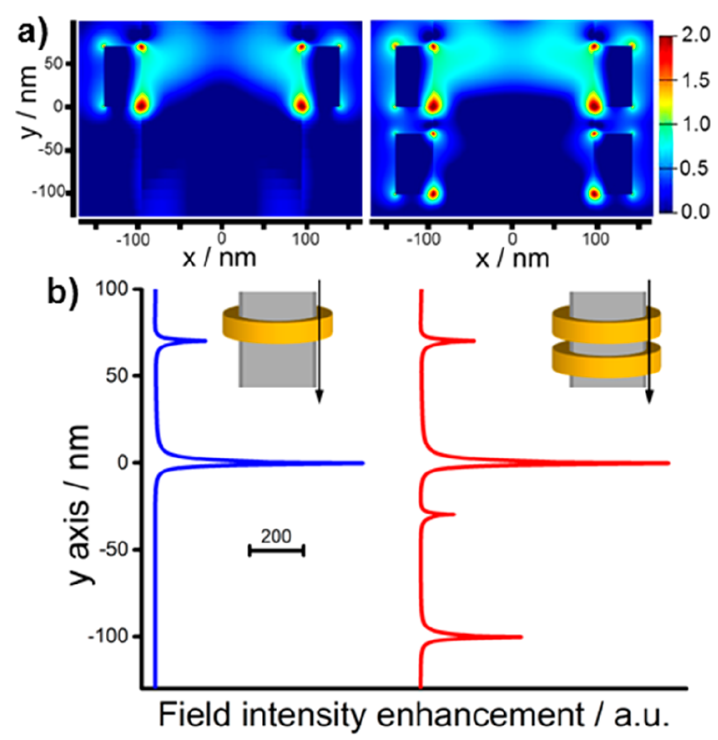

Figure 4. FDTD simulations. (a) E-field intensity enhancement maps at the laser excitation wavelength (i.e., $785 \mathrm{~nm}$ ) for a single $\mathrm{Au}$ ring (left) and a $\mathrm{Au}$ ring dimer (30 nm gap, right) around a Si nanowire in air. The ring region only is shown for clarity. The ring dimensions are $70 \mathrm{~nm}$ height, $190 \mathrm{~nm}$ inner diameter, and $45 \mathrm{~nm}$ thickness. Both single rings and ring dimers are located at the middle of the Si wire (i.e., at $1.5 \mu \mathrm{m}$ from the bottom of the wire). The bottom end of the top ring correspond to the position $y=0$. The plane wave source propagates along the $y$ axis (from top to bottom), and the electric field is polarized along the $x$ axis. The logarithmic color scale of field intensity enhancement is the same for both maps. (b) E-field intensity enhancement line scan at the surface of the nanowire $(1 \mathrm{~nm}$ distance from the surface) in the ring region, schematically depicted by the black arrows. Left: single ring (blue curve). Right: ring dimer (red curve), linear scale. The line scans are offset for clarity. excitation wavelength originates from the $\mathrm{BDT}$ molecules that are adsorbed in the hot spot regions, located near the $\mathrm{Si}-\mathrm{Au}$ interface (Figure 4a), we would expect the $\mathrm{Au}$ ring dimer sample to provide a Raman signal approximately 1.6 times larger than the signal measured at the single $\mathrm{Au}$ ring sample. Experimentally, we measured an increase in the Raman signal of $\sim 1.8$ between the two samples, which matches the simulations. Our simulations also suggest that the Au rings concentrate the light within specific parts of the Si nanowires (Figure S19): the E-field intensity in the Si located above the rings (top $1.5 \mu \mathrm{m}$ ) is increased by a factor of $\sim 2$ for a single $\mathrm{Au}$ ring and $\sim 3$ for a $\mathrm{Au}$ ring dimer compared to a pristine $\mathrm{Si}$ nanowire, while it is nearly suppressed below the rings (bottom $1.5 \mu \mathrm{m}$ of the Si wire). These results demonstrate the reliability and potential of the method to engineer plasmonic fields and tune light absorption in three dimensions within Si wire arrays over large areas.

To conclude, we demonstrate the synthesis of complex hybrid c-Si@metal micro- and nanowire architectures. The method is based on sacrificial $\mathrm{SiO}_{2}$ coating and the formation of a porous membrane with tunable dimensions, which serves as a template for the electrochemical deposition of metal shells around the Si nanowires. Defined ring architectures can be produced by selective etching of the sacrificial metal shells. This is a shift in paradigm for templated syntheses that have, until now, relied on the direct replication of a template structure. $^{54}$ 3DEAL can be used to pattern well-defined preexisting three-dimensional nanostructures with metal features as small as $5 \mathrm{~nm}$. The technique is compatible with unconventional morphologies such as kinked nanowires and could be easily generalized to a variety of $\mathrm{Si}$ nanostructures with, for example, different cross-section morphologies ${ }^{45}$ or conical shapes. ${ }^{55}$ The remarkably small standard deviation obtained from Raman measurements underlines the high uniformity of the hybrid structures and demonstrates the reliability of the technique to control and shape electromagnetic near-fields in three dimensions. Our simulation show that this could be used to spatially control light absorption. The current experimental design requires a metal film at the bottom of the wire array, which is conveniently present after MACE. For this reason, our approach should be compatible with other important semiconductors that can be nanostructured via MACE such as GaAs or InP. ${ }^{56,57}$ Because it is possible to selectively deposit a continuous and conductive metal film at the bottom of "bare" Si microwire arrays (i.e., without a metal base layer in the first place), ${ }^{58}$ we believe that 3DEAL will be also compatible with other types of $\mathrm{Si}$ wire arrays made, for example, via vapor-liquid-solid growth ${ }^{19}$ or photolithography and dry-etching processes. ${ }^{21}$ Ultimately, our work is a proof-of-concept of a universal benchtop approach for the patterning of three-dimensional nanostructured architectures that have a continuously open pore system.

\section{ASSOCIATED CONTENT}

\section{S Supporting Information}

The Supporting Information is available free of charge on the ACS Publications website at DOI: 10.1021/acs.nanolett.8b03608.

A list of the Si@metal structure dimensions, additional details on materials and methods, SEM and STEM images, EDX and Raman maps, and FDTD simulation results $(\mathrm{PDF})$ 


\section{AUTHOR INFORMATION}

\section{Corresponding Author}

*E-mail: gilles.bourret@sbg.ac.at.

\section{ORCID}

Maurizio Musso: 0000-0001-6631-5206

Nicolas Vogel: 0000-0002-9831-6905

Oliver Diwald: 0000-0002-2425-5281

Gilles R. Bourret: 0000-0002-9774-1686

\section{Author Contributions}

The manuscript was written through contributions of all authors. All authors have given approval to the final version of the manuscript.

\section{Funding}

O.D., G.R.B., and F.J.W. gratefully acknowledge support from the Austrian Science Fund FWF for project no. P-28797 and the Allergy-Cancer-BioNano (ACBN) research initiative from the University of Salzburg. M.M., G.R.B., and A.R. gratefully acknowledge financial support provided by the European Regional Development Fund and Interreg V-A Italy-Austria 2014-2020 through the Interreg Italy-Austria project ITAT 1023 InCIMA (http://www.elettra.eu/Prj/InCIMa/). M.R. and N.V. acknowledge support by the Deutsche Forschungsgemeinschaft (DFG) under grant no. VO1824/6-1. N.V. also acknowledges support by the Interdisciplinary Center for Functional Particle Systems (FPS). We thank Grant Osborne for his help with the $3 \mathrm{D}$ printer in preparing the sample holders used for MACE.

\section{Notes}

The authors declare no competing financial interest.

\section{REFERENCES}

(1) Kelzenberg, M. D.; Boettcher, S. W.; Petykiewicz, J. A.; TurnerEvans, D. B.; Putnam, M. C.; Warren, E. L.; Spurgeon, J. M.; Briggs, R. M.; Lewis, N. S.; Atwater, H. A. "Enhanced absorption and carrier collection in Si wire arrays for photovoltaic applications". Nat. Mater. 2010, 9, 239-244.

(2) Dasgupta, N. P.; Sun, J.; Liu, C.; Brittman, S.; Andrews, S. C.; Lim, J.; Gao, H.; Yan, R.; Yang, P. "25th Anniversary Article: Semiconductor Nanowires - Synthesis, Characterization, and Applications". Adv. Mater. 2014, 26, 2137-2184.

(3) Länk, N. O.; Verre, R.; Johansson, P.; Käll, M. Large-Scale Silicon Nanophotonic Metasurfaces with Polarization Independent Near-Perfect Absorption. Nano Lett. 2017, 17, 3054-3060.

(4) Su, Y.; Liu, C.; Brittman, S.; Tang, J.; Fu, A.; Kornienko, N.; Kong, Q.; Yang, P. "Single-Nanowire Photoelectrochemistry". Nat. Nanotechnol. 2016, 11, 609-612.

(5) Vijselaar, W.; Westerik, P.; Veerbeek, J.; Tiggelaar, R. M.; Berenschot, E.; Tas, N. R.; Gardeniers, H.; Huskens, J. "Spatial decoupling of light absorption and catalytic activity of Ni-Mo-loaded high-aspect-ratio silicon microwire photocathodes". Nat. Energy 2018, $3,185-192$

(6) Wu, Y.; Xiang, J.; Yang, C.; Lu, W.; Lieber, C. M. "Single-crystal metallic nanowires and metal/semiconductor nanowire heterostructures". Nature 2004, 430, 61-65.

(7) Glassner, S.; Zeiner, C.; Periwal, P.; Baron, T.; Bertagnolli, E.; Lugstein, A. "Multi-mode silicon nanowire transistors". Nano Lett. 2014, 14, 6699-6703.

(8) Caldarola, M.; Albella, P.; Cortés, E.; Rahmani, M.; Roschuk, T.; Grinblat, G.; Oulton, R. F.; Bragas, A. V.; Maier, S. A. "Nonplasmonic nanoantennas for surface enhanced spectroscopies with ultra-low heat conversion". Nat. Commun. 2015, 6, 7915.

(9) Chan, C. K.; Peng, H.; Liu, G.; McIlwrath, K.; Zhang, X. F.; Huggins, R. A.; Cui, Y. "High Performance Lithium Battery Anodes Using Silicon Nanowires". Nat. Nanotechnol. 2008, 3, 31-35.
(10) Liu, C.; Tang, J.; Chen, H. M.; Liu, B.; Yang, P. "A Fully Integrated Nanosystem of Semiconductor Nanowires for Direct Solar Water Splitting". Nano Lett. 2013, 13, 2989.

(11) O’Brien, P. G.; Sandhel, A.; Wood, T. E.; Jelle, A. A.; Hoch, L. B.; Perovic, D. D.; Mims, C. A.; Ozin, G. A. "Photomethanation of Gaseous CO2 over Ru/Silicon Nanowire Catalysts with Visible and Near-Infrared Photons". Adv. Sci. 2014, 1, 1400001.

(12) Agarwal, D.; Aspetti, C. O.; Cargnello, M.; Ren, M.; Yoo, J.; Murray, C. B.; Agarwal, R. "Engineering Localized Surface Plasmon Interactions in Gold by Silicon Nanowire for Enhanced Heating and Photocatalysis". Nano Lett. 2017, 17, 1839-1845.

(13) Garnett, E.; Yang, P. "Light Trapping in Silicon Nanowire Solar Cells". Nano Lett. 2010, 10, 1082-1087.

(14) Liu, R.; Chen, R.; Elthakeb, A. T.; Lee, S. H.; Hinckley, S.; Khraiche, M. L.; Scott, J.; Pre, D.; Hwang, Y.; Tanaka, A.; Ro, Y. G.; Matsushita, A. K.; Dai, X.; Soci, C.; Biesmans, S.; James, A.; Nogan, J.; Jungjohann, K. L.; Pete, D. V.; Webb, D. B.; Zou, Y.; Bang, A. G.; Dayeh, S. A. "High Density Individually Addressable Nanowire Arrays Record Intracellular Activity from Primary Rodent and Human Stem Cell Derived Neurons". Nano Lett. 2017, 17, 2757-2764.

(15) Cho, C.-H.; Aspetti, C. O.; Park, J.; Agarwal, R. "Silicon coupled with plasmon nanocavity generates bright visible hotluminescence". Nat. Photonics 2013, 7, 285-289.

(16) Wang, X.; Peng, K.-Q.; Hu, Y.; Zhang, F.-Q.; Hu, B.; Li, L.; Wang, M.; Meng, X.-M.; Lee, S.-T. "Silicon/Hematite Core/Shell Nanowire Array Decorated with Gold Nanoparticles for Unbiased Solar Water Oxidation". Nano Lett. 2014, 14, 18-23.

(17) Liu, D.; Yang, D.; Gao, Y.; Ma, J.; Long, R.; Wang, C.; Xiong, Y. "Flexible Near-Infrared Photovoltaic Devices Based on Plasmonic Hot-Electron Injection into Silicon Nanowire Arrays". Angew. Chem. 2016, 128, 4653-4657.

(18) Maruo, S.; Fourkas, J. T. "Recent progress in multiphoton microfabrication". Laser Photonics Rev. 2008, 2, 100-111.

(19) Lauhon, L. J.; Gudiksen, M. S.; Wang, D.; Lieber, C. M. "Epitaxial Core-Shell and Core-Multi-Shell Nanowire Heterostructures". Nature 2002, 420, 57-61.

(20) Milbrat, A.; Elbersen, R.; Kas, R.; Tiggelaar, R. M.; Gardeniers, H.; Mul, G.; Huskens, J. "Spatioselective Electrochemical and Photoelectrochemical Functionalization of Silicon Microwires with Axial p/n Junctions". Adv. Mater. 2016, 28, 1400-1405.

(21) Ozel, T.; Zhang, B. A.; Gao, R.; Day, R. W.; Lieber, C. M.; Nocera, D. G. "Electrochemical Deposition of Conformal and Functional Layers on High Aspect Ratio Silicon Micro/Nanowires". Nano Lett. 2017, 17, 4502-4507.

(22) Mankin, M. N.; Day, R. W.; Gao, R.; No, Y.-S.; Kim, S.-K.; McClelland, A. A.; Bell, D. C.; Park, H.-G.; Lieber, C. M. "FacetSelective Epitaxy of Compound Semiconductors on Faceted Silicon Nanowires". Nano Lett. 2015, 15, 4776-4782.

(23) Day, R. W.; Mankin, M. N.; Gao, R.; No, Y.-S.; Kim, S.-K.; Bell, D. C.; Park, H.-G.; Lieber, C. M. "Plateau-Rayleigh crystal growth of periodic shells on one-dimensional substrates". Nat. Nanotechnol. 2015, 10, 345-352.

(24) Christesen, J. D.; Pinion, C. W.; Grumstrup, E. M.; Papanikolas, J. M.; Cahoon, J. F. "Synthetically Encoding $10 \mathrm{~nm}$ Morphology in Silicon Nanowires". Nano Lett. 2013, 13, 6281-6286.

(25) Luo, Z.; Jiang, Y.; Myers, B. D.; Isheim, D.; Wu, J.; Zimmerman, J. F.; Wang, Z.; Li, Q.; Wang, Y.; Chen, X.; Dravid, V. P.; Seidman, D. N.; Tian, B. "Atomic Gold-Enabled ThreeDimensional Lithography for Silicon Mesostructures". Science 2015, $348,1451-1455$.

(26) Nguyen, N. T.; Altomare, M.; Yoo, J.; Schmuki, P. "Efficient Photocatalytic H 2 Evolution: Controlled Dewetting-Dealloying to Fabricate Site-Selective High-Activity Nanoporous Au Particles on Highly Ordered TiO2 Nanotube Arrays". Adv. Mater. 2015, 27, $3208-3215$

(27) Kelly, K. L.; Coronado, E.; Zhao, L. L.; Schatz, G. C. "The Optical Properties of Metal Nanoparticles: The Influence of Size, Shape, and Dielectric Environment". J. Phys. Chem. B 2003, 107, $668-677$. 
(28) Pelton, M.; Aizpurua, J.; Bryant, G. "Metal-nanoparticle plasmonics". Laser Photonics Rev. 2008, 2, 136-159.

(29) Christopher, P.; Xin, H.; Linic, S. "Visible light enhanced catalytic oxidation reactions on plasmonic Ag nanostructures". Nat. Chem. 2011, 3, 467-472.

(30) Bourret, G. R.; Ozel, T.; Blaber, M.; Shade, C.; Schatz, G. C.; Mirkin, C. A. " Long-Range Plasmophore Rulers". Nano Lett. 2013, 13, 2270-2275.

(31) Osberg, K. D.; Rycenga, M.; Bourret, G. R.; Brown, K.; Mirkin, C. A. "Dispersible Surface-Enhanced Raman Scattering Nanosheets". Adv. Mater. 2012, 24, 6065-6070.

(32) Ozel, T.; Bourret, G. R.; Schmucker, A.; Brown, K.; Mirkin, C. A. "Hybrid Semiconductor Core-Shell Nanowires with Tunable Plasmonic Nanoantennas". Adv. Mater. 2013, 25, 4515-4520.

(33) Ozel, T.; Bourret, G. R.; Mirkin, C. A. "Coaxial Lithography". Nat. Nanotechnol. 2015, 10, 319-324.

(34) Schlücker, S. "Surface-Enhanced Raman Spectroscopy: Concept and Chemical Applications". Angew. Chem., Int. Ed. 2014, 53, 4756-4795.

(35) Brongersma, M. L.; Halas, N. J.; Nordlander, P. "Plasmoninduced hot carrier science technology". Nat. Nanotechnol. 2015, 10, 25-34.

(36) Reyer, A.; Prinz, A.; Giancristofaro, S.; Schneider, J.; Menezes, D. B.; Zickler, G.; Bourret, G. R.; Musso, M. E. "Investigation of Mass-Produced Substrates for Reproducible Surface-Enhanced Raman Scattering Measurements over Large Areas". ACS Appl. Mater. Interfaces 2017, 9, 25445-25454.

(37) Itoh, T.; Yamamoto, Y. S.; Ozaki, Y. "Plasmon-enhanced spectroscopy of absorption and spontaneous emissions explained using cavity quantum optics". Chem. Soc. Rev. 2017, 46, 3904-3921.

(38) Penner, R. M.; Martin, C. R. "Preparation and Electrochemical Characterization of Ultramicroelectrode Ensembles". Anal. Chem. 1987, 59, 2625-2630.

(39) Martin, C. R. "Nanomaterials: A Membrane-Based Synthetic Approach". Science 1994, 266, 1961-1966.

(40) Routkevitch, D.; Bigioni, T.; Moskovits, M.; Xu, J. M. "Electrochemical fabrication of CdS nanowire arrays in porous anodic aluminum oxide templates". J. Phys. Chem. 1996, 100, 14037-14047. (41) Vogel, N.; Weiss, C. K.; Landfester, K. "From soft to hard: the generation of functional and complex colloidal monolayers for nanolithography". Soft Matter 2012, 8, 4044-4061.

(42) Vogel, N.; Retsch, M.; Fustin, C.-A.; del Campo, A.; Jonas, U. "Advances in Colloidal Assembly: The Design of Structure and Hierarchy in Two and Three Dimensions". Chem. Rev. 2015, 115, 6265-6311.

(43) Huang, Z.; Fang, H.; Zhu, J. "Fabrication of Silicon Nanowire Arrays with Controlled Diameter, Length, and Density". Adv. Mater. 2007, 19, 744-748.

(44) Huang, Z.; Geyer, N.; Werner, P.; de Boor, J.; Gösele, U. "Metal-Assisted Chemical Etching of Silicon: A Review". Adv. Mater. 2011, 23, 285-308.

(45) Wendisch, F. J.; Oberreiter, R.; Salihovic, M.; Elsaesser, M. S.; Bourret, G. R. "Confined Etching within 2D and 3D Colloidal Crystals for Tunable Nanostructured Templates: Local Environment Matters". ACS Appl. Mater. Interfaces 2017, 9, 3931-3939.

(46) Vogel, N.; Goerres, S.; Landfester, K.; Weiss, C. K. "A Convenient Method to Produce Close- and Non-close-Packed Monolayers using Direct Assembly at the Air-Water Interface and Subsequent Plasma-Induced Size Reduction". Macromol. Chem. Phys. 2011, 212, 1719-1734.

(47) Ozel, T.; Ashley, M. J.; Bourret, G. R.; Ross, M. B.; Schatz, G. C.; Mirkin, C. A. "Solution-Dispersible Metal Nanorings with Deliberately Controllable Compositions and Architectural Parameters for Tunable Plasmonic Response". Nano Lett. 2015, 15, 5273-5278.

(48) Miao, Z.; Xu, D.; Ouyang, J.; Guo, G.; Zhao, X.; Tang, Y. "Electrochemically Induced Sol-Gel Preparation of Single-Crystalline TiO2 Nanowires". Nano Lett. 2002, 2, 717-720.

(49) Tena-Zaera, R.; Elias, J.; Wang, G.; Levy-Clement, C. "Role of Chloride Ions on Electrochemical Deposition of $\mathrm{ZnO}$ Nanowire
Arrays from O2 Reduction". J. Phys. Chem. C 2007, 111, 1670616711.

(50) Chen, Y.; Li, L.; Zhang, C.; Tuan, C.-C.; Chen, X.; Gao, J.; Wong, C.-P. "Controlling Kink Geometry in Nanowires Fabricated by Alternating Metal-Assisted Chemical Etching". Nano Lett. 2017, 17, 1014-1019.

(51) Nagpal, P.; Lindquist, N. C.; Oh, S.-H.; Norris, D. J. "Ultrasmooth Patterned Metals for Plasmonics and Metamaterials". Science 2009, 325, 594-597.

(52) Uchinokura, K.; Sekine, T.; Matsuura, E. "Raman Scattering by Silicon". Solid State Commun. 1972, 11, 47-49.

(53) Joo, S. W.; Han, S. W.; Kim, K. "Adsorption of 1,4Benzenedithiol on Gold and Silver Surfaces: Surface-Enhanced Raman Scattering Study". J. Colloid Interface Sci. 2001, 240, 391-399.

(54) Jones, M. R.; Osberg, K. D.; Macfarlane, R. J.; Langille, M. R.; Mirkin, C. A. "Templated techniques for the synthesis and assembly of plasmonic nanostructures". Chem. Rev. 2011, 111, 3736-3827.

(55) Lin, H.; Cheung, H.-Y.; Xiu, F.; Wang, F.; Yip, S.; Han, N.; Hung, T.; Zhou, J.; Ho, J. C.; Wong, C.-Y. "Developing controllable anisotropic wet etching to achieve silicon nanorods, nanopencils and nanocones for efficient photon trapping". J. Mater. Chem. A 2013, 1, 9942-9946.

(56) DeJarld, M.; Shin, J. C.; Chern, W.; Chanda, D.; Balasundaram, K.; Rogers, J. A.; Li, X. "Formation of High Aspect Ratio GaAs Nanostructures with Metal-Assisted Chemical Etching". Nano Lett. 2011, 11, 5259-5263.

(57) Kim, S. H.; Mohseni, P. K.; Song, Y.; Ishihara, T.; Li, X. "Inverse Metal-Assisted Chemical Etching Produces Smooth High Aspect Ratio InP Nanostructures". Nano Lett. 2015, 15, 641-648.

(58) Xiang, C.; Meng, A. C.; Lewis, N. S. "Evaluation and optimization of mass transport of redox species in silicon microwirearray photoelectrodes". Proc. Natl. Acad. Sci. U. S. A. 2012, 109, $15622-15627$. 